Research Article

\title{
Performance Analysis of Data Transmission for Joint Radar and Communication Systems
}

\author{
Jianhua Lu $\mathbb{D},{ }^{1,2}$ Tuanwei Tian, ${ }^{1}$ Yanli Tang, ${ }^{1}$ and Bin Tang $\mathbb{D}^{1}$ \\ ${ }^{1}$ School of Information and Communication Engineering, University of Electronic Science and Technology of China, \\ Chengdu 611731, China \\ ${ }^{2}$ School of Air Operations and Services, Aviation University of Air Force, Changchun 130022, China \\ Correspondence should be addressed to Bin Tang; bint@uestc.edu.cn
}

Received 30 November 2020; Revised 7 January 2021; Accepted 27 February 2021; Published 16 March 2021

Academic Editor: Fangqing Wen

Copyright (C) 2021 Jianhua Lu et al. This is an open access article distributed under the Creative Commons Attribution License, which permits unrestricted use, distribution, and reproduction in any medium, provided the original work is properly cited.

\begin{abstract}
This paper investigates the problem of data transmission for the joint radar and communication systems (JRCSs). The performance of the JRCS is characterized by data throughput related to the radar echo data (RED) and communication data rate (CDR). Two spectral coexistence schemes are proposed based on the degree of spectrum sharing for radar and communication, i.e., the isolated subfrequency band (ISFB) and mix-used frequency band (MUFB) schemes. Firstly, the signals of radar and communication are operated on the isolated subcarriers, enabling the received signals to be processed independently and bringing the advantage of interference avoidance. Secondly, the signals of radar and communication can be jointly operated on the same subcarriers for the MUFB scheme, which enhances the spectrum efficiency. Unlike the ISFB scheme, the CDR of the MUFB scheme is maximized along with the interference from the radar signal, and meanwhile, the allocated radar power on each subcarrier is derived by maximizing the radar mutual information. Numerical results show that the MUFB scheme significantly improves the performance of data transmission over the ISFB scheme, and a significant performance gain in the data transmission can be achieved, compared to the average power allocation case.
\end{abstract}

\section{Introduction}

Recent information battlefield scenarios urge modern radar equipment to deploy optimally $[1,2]$ and exchange the estimation information [3] with each other. How to form an integrated system by combining the radar equipment with the communication equipment and solve the problems of the generalization, such as tremendous wireless data traffic, rational use of the system resources [4-6] has been the theme of increasing research over recent years. Spectrum sharing between radar and communication systems, which can overcome the shortage of spectrum resources and improve the efficiency of the spectrum significantly, is one of the essential research studies and has been a hot topic [7-11]. One factor that has to be emphasized, however, is the interference concerns caused by the cochannel spectrum sharing of radar and communication systems [12].
Two directions of coexistence between radar and communication have been developed:

(i) The first one aims to develop a dual-function system that simultaneously realizes radar and communication functions [7, 11, 13-19]. In [13], a method of utilizing an orthogonal frequency division multiplexing (OFDM) signal to operate the functions of radar and communication systems is applied to the problem of radar and communication coexistence. The similar strategy can also found in $[14,15,20]$. Embed communication information into the radar waveform so that radar and communication functions can be realized by one common signal. This dual-function radar and communication (DFRC) technique includes sidelobe amplitude modulation (AM) scheme [16], waveform diversity-based 
scheme [17], phase shift keying (PSK) scheme [18], and multiwaveform amplitude shift keying (ASK) scheme [19].

(ii) The second direction focuses on the spectral coexistence between radar and communication systems. The authors in [21] present the fundamental spectrumsharing concepts and technologies. An overview of interference mitigation techniques is introduced in [22]. In [23], radar and communication systems are operated at the same frequency band using cognitive radio technology, to mitigate interference between the two systems. The authors in $[24,25]$ derive the achievable performance bounds of coexistence between radar and communication systems. A novel parameter, named estimation information rate, is proposed to measure the performance of radar systems and data transmission rate for the communication systems. In [26], to minimize the interference caused by spectrum sharing between radar and communication systems, the null-space projection (NSP) method is adopted. An IEEE 802.11ad-based radar is proposed in [27], which enables a joint waveform for both automotive radar and $\mathrm{mm}$-wave communication systems. For the situation with unreliable radar estimation, the authors in [28] propose a system level interference cancellation algorithm. In [29], spectrum sharing between radar and communication based on codesign transmitted waveform is studied to minimize the effective interference from each other. Two generalized sidelobe cancellers are used in [30] to increase the communication data rate without affecting the radar effectiveness. The authors in [31] analyze the problem of power minimization-based radar waveform design considering the existence of communication signals on the same band. The problem of power allocation between multicarrier radar and communication systems is emphasized in $[32,33]$. Based on the information theory, the authors in [34] design an adaptive signal for the integrated OFDM radar and communication system.

To the best of our knowledge, the performance of data transmission for the joint radar and communication systems (JRCS) has not been mentioned in the above works. This is indeed the main topic of this paper. The contributions are as follows:

(1) Based on the degree of spectrum sharing for radar and communication, we present two spectral coexistence schemes, i.e., the isolated subfrequency band (ISFB) and mix-used frequency band (MUFB) schemes

(2) Under certain range resolution $\Delta R$ and scanning speed $f_{\theta}$, we give the amount of radar echo data (RED) and the expressions of data throughput for the ISFB and MUFB schemes, which maximize the data throughput under a certain range resolution and scanning speed along with a total transmit power constraint, are proposed

(3) Choosing the maximization of data throughput along with a total transmit power constraint as optimization, we maximize the data throughput of the JRCS and drive the closed-form solution of the power allocation

This paper is organized as follows. The received signals at radar and communication receivers are modeled in Section 2. The performances of data transmission for the ISFB and MUFB schemes are analyzed in Section 3 and Section 4, respectively. Section 5 gives a numerical example, and conclusions are drawn in Section 6.

Notations: $C$ denotes the set complex. $C^{N}\left(C^{N \times N}\right)$ denotes the set of $N \times 1$ vector $(N \times N$ matrix $)$ with entries from $C .(\cdot)^{*},(\cdot)^{T}$, and $(\cdot)^{H}$ denote the optimality, transpose, and complex conjugate transpose, respectively. The symbol $\operatorname{det}(\cdot)$ indicates the determinant of a square matrix. $\operatorname{diag}\{\cdot\}$ is the diagonal matrix with all elements on the main diagonal. The complex Gaussian distribution is denoted by $\mathscr{C} N(\cdot, \cdot)$. Finally, $(x, 0)^{+}$represents the positive part of $x$.

\section{System Model}

As shown in Figure 1, we consider such a scenario that an integrated transmitter/receiver scans the region of interest and then transmits the scanned information to the communication receiver.The integrated transmitter consists of $M_{r}$ antennas for transmitting radar signal, $M_{c}$ antennas for transmitting communication signal, and $N_{r}$ antennas for receiving the reflected radar signal. The received signal at the communication receiver with $N_{c}$ antennas is a compound of signals scattered from the integrated transmitter and region of interest. Both radar and communication signals are assumed to be the OFDM-type multicarrier signals with $K$ subcarriers. Moreover, the frequency spacing $\Delta f$ among the $K$ subcarriers is assumed to be sufficiently large. Perfect OFDM symbol timing at the receiver is assumed, and the cyclic prefix (CP) of each received signal is removed before OFDM demodulation. Without loss of generality, we assume $M_{r}=M_{c}=M, N_{r}=N_{c}=N$ in this paper.

2.1. Radar Receiver. Define $\widetilde{\mathbf{S}}(k)=\left[\widetilde{s}_{1}(k), \widetilde{s}_{2}(k), \ldots, \widetilde{s}_{M}(k)\right]$ as the transmitted radar waveform on the $k$-th subcarrier, and assume $K>M ; K>N$. Let $\widetilde{\mathbf{y}}_{n}=\left[\widetilde{y}_{n}(1), \widetilde{y}_{n}(2), \ldots\right.$, $\left.\tilde{y}_{n}(K)\right]$ be the received signal of the $n$-th receive antenna, then we have

$$
\widetilde{\mathbf{y}}_{n}=\widetilde{\mathbf{h}}_{n}^{T} \widetilde{\mathbf{S}}^{T}+\widetilde{\mathbf{w}}_{n},
$$

where $\widetilde{\mathbf{h}}_{n}=\left[\widetilde{h}_{1, n}, \widetilde{h}_{2, n}, \ldots, \widetilde{h}_{M, n}\right]^{T}$ are the target response from the $m$-th integrated transmitter to the $n$-th receiver, $\widetilde{\mathbf{S}} \in C^{K \times M}$ is the transmitted waveform matrix with $\widetilde{\mathbf{s}}=[\widetilde{\mathbf{s}}(1), \widetilde{\mathbf{s}}(2), \ldots, \widetilde{\mathbf{s}}(K)]^{T}, \widetilde{\mathbf{w}}_{n} \in C^{1 \times K}$ is the noise vector, and $\widetilde{\mathbf{w}}_{n}=\left[\widetilde{w}_{n}(1), \widetilde{w}_{n}(2), \ldots, \widetilde{w}_{n}(K)\right]$.

Let $\widetilde{\mathbf{Y}}=\left[\widetilde{\mathbf{y}}_{1}^{T}, \widetilde{\mathbf{y}}_{2}^{T}, \ldots, \widetilde{\mathbf{Y}}_{N}^{T}\right]$, then $\widetilde{\mathbf{Y}} \in C^{K \times N}$ can be given by

$$
\widetilde{\mathbf{Y}}=\widetilde{\mathbf{S}} \widetilde{\mathbf{H}}+\widetilde{\mathbf{W}} \text {, }
$$



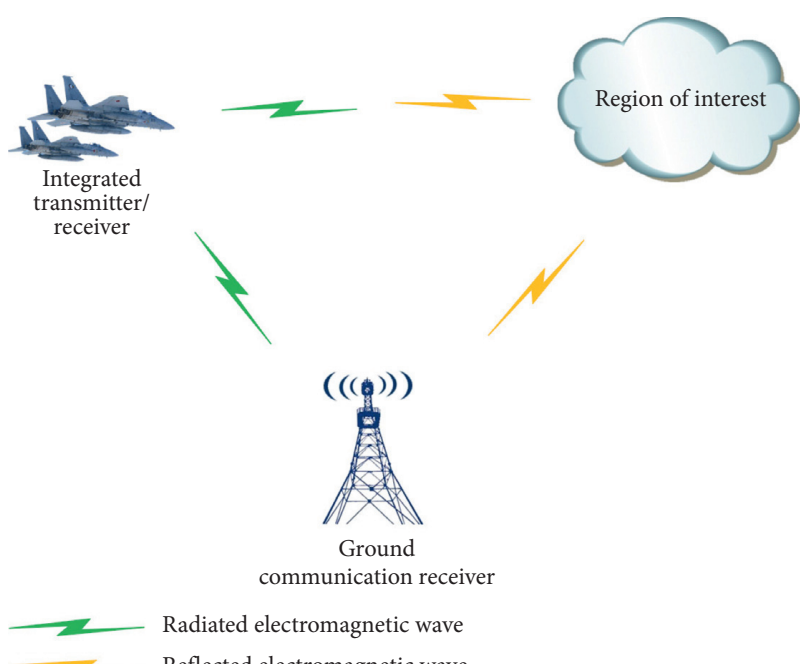

Reflected electromagnetic wave

FIgURE 1: The integrated transmit and receive system communicates with the communication receiver on the ground while detecting the region of interest.

where $\widetilde{\mathbf{H}} \in C^{M \times N}$ is the target scattering matrix, $\widetilde{\mathbf{H}}=\left[\widetilde{\mathbf{h}}_{1}, \widetilde{\mathbf{h}}_{2}, \ldots, \widetilde{\mathbf{h}}_{N}\right], \widetilde{\mathbf{W}} \in C^{K \times N}$ is the noise matrix, and $\widetilde{\mathbf{W}}=\left[\widetilde{\mathbf{w}}_{1}^{T}, \widetilde{\mathbf{w}}_{2}^{T}, \ldots, \widetilde{\mathbf{w}}_{N}^{T}\right]$. For simplicity, we assume the columns of $\widetilde{\mathbf{H}}$ and $\widetilde{\mathbf{W}}$ are independent and identically distributed (i.i.d), with distribution $\square \square\left(0, \widetilde{\Sigma}_{H}\right)$ and $\square \square\left(0, \widetilde{\Sigma}_{\mathbf{w}}\right)$, respectively.

Radar mutual information has been used as a parameter in many scenarios to characterize the radar system [32, 35-37]. The more radar mutual information is, the better performance of radar is, which can be derived from the mutual information between the input $\widetilde{\mathbf{S}}$ and output $\widetilde{\mathbf{Y}}$, namely,

$$
\begin{aligned}
I(\widetilde{\mathbf{S}} ; \widetilde{\mathbf{Y}})= & h(\widetilde{\mathbf{Y}})-h(\widetilde{\mathbf{Y}} \mid \widetilde{\mathbf{S}})=\log _{2} \operatorname{det}\left(\widetilde{\mathbf{S}} \widetilde{\Sigma}_{H} \widetilde{\mathbf{S}}^{H}+\widetilde{\Sigma}_{\mathbf{w}}\right) \\
& -\log _{2} \operatorname{det}\left(\widetilde{\Sigma}_{\mathbf{w}}\right),
\end{aligned}
$$

where $h(\cdot)$ denotes the differential entropy of a random variable.

2.2. Communication Receiver. Let $\mathbf{s}(k)=\left[s_{1}(k), s_{2}(k), \ldots\right.$, $\left.s_{M}(k)\right]$ be the transmitted communication waveform on the $k$-th subcarrier and $\mathbf{y}_{n}=\left[y_{n}(1), y_{n}(2), \ldots, y_{n}(K)\right]$ be the received signal of the $n$-th communication antenna, then we have

$$
\mathbf{y}_{n}=\mathbf{h}_{n}^{T} \mathbf{S}^{T}+\mathbf{h}_{r, n}^{T} \widetilde{\mathbf{S}}^{T}+\mathbf{w}_{n}
$$

where $\mathbf{h}_{n}=\left[h_{1, n}, h_{2, n}, \ldots, h_{M, n}\right]^{T}$ are the channel responses from the $m$-th integrated transmitter to the $n$-th communication receiver, $\mathbf{h}_{r, n}=\left[h_{1, r, n}, h_{2, r, n}, \ldots, h_{M, r, n}\right]^{T}$ are the target response of link from the $m$-th integrated transmitter to the region of interest and to the $n$-th communication antenna, $\mathbf{S} \in C^{K \times M}$ is the transmitted communication waveform matrix, $\mathbf{S}=[\mathbf{s}(1), \mathbf{s}(2), \ldots, \mathbf{s}(K)]^{T}, \mathbf{w} \in C^{1 \times K}$ is the noise vector at the communication receiver, and $\mathbf{w}=[w(1), w(2), \ldots, w(K)]$.

Let $\mathbf{Y}=\left[\mathbf{y}_{1}^{T}, \mathbf{y}_{2}^{T}, \ldots, \mathbf{y}_{N}^{T}\right]$, then $\mathbf{Y} \in C^{K \times N}$ can be given by

$$
\mathbf{Y}=\mathbf{S H}+\widetilde{\mathbf{S}} \mathbf{H}_{r}+\mathbf{W},
$$

where $\mathbf{H} \in C^{M \times N}$ is the channel response matrix, $\mathbf{H}=\left[\mathbf{h}_{1}, \mathbf{h}_{2}, \ldots, \mathbf{h}_{N}\right], \mathbf{H}_{r} \in C^{M \times N}$ is the target response matrix, $\mathbf{H}_{r}=\left[\mathbf{h}_{1, r}, \mathbf{h}_{2, r}, \ldots, \mathbf{h}_{N, r}\right], \mathbf{W} \in C^{K \times N}$ is the noise matrix, and $\mathbf{W}=\left[\mathbf{w}_{1}^{T}, \mathbf{w}_{2}^{T}, \ldots, \mathbf{w}_{N}^{T}\right]$. For simplicity, we assume that the columns of $\mathbf{H}, \mathbf{H}_{r}$, and $\mathbf{W}$ are i.i.d with distributions $\mathscr{C} \mathcal{N}(0, \Sigma), \quad \mathscr{C} \mathscr{N}\left(0, \Sigma_{r}\right)$, and $\mathscr{C} \mathscr{N}\left(0, \Sigma_{\text {w }}\right)$, respectively.

The mutual information between the input $\mathbf{S}$ and output $\mathbf{Y}$ is given by

$$
\begin{aligned}
I(\mathbf{S} ; \mathbf{Y})= & h(\mathbf{Y})-h(\mathbf{Y} \mid \mathbf{S}) \\
= & h(\mathbf{Y})-h\left(\widetilde{\mathbf{S}} \mathbf{H}_{r}+\mathbf{W}\right) \\
= & \log _{2} \operatorname{det}\left(\mathbf{S} \Sigma \mathbf{S}^{H}+\widetilde{\mathbf{S}} \Sigma_{r} \widetilde{\mathbf{S}}^{H}+\Sigma_{\mathbf{w}}\right) \\
& -\log _{2} \operatorname{det}\left(\widetilde{\mathbf{S}} \Sigma_{r} \widetilde{\mathbf{S}}^{H}+\Sigma_{\mathbf{w}}\right)
\end{aligned}
$$

Thus, the communication data rate can be given by

$$
\begin{aligned}
\mathscr{C}= & K_{c} \Delta f\left(\log _{2} \operatorname{det}\left(\mathbf{S} \Sigma \mathbf{S}^{H}+\widetilde{\mathbf{S}} \Sigma_{r} \widetilde{\mathbf{S}}^{H}+\Sigma_{\mathbf{w}}\right)\right. \\
& \left.-\log _{2} \operatorname{det}\left(\widetilde{\mathbf{S}} \Sigma_{r} \widetilde{\mathbf{S}}^{H}+\Sigma_{\mathbf{w}}\right)\right)\left(\frac{\text { bit }}{\mathrm{s}}\right),
\end{aligned}
$$

where $K_{c}$ denotes the number of communication subcarrier.

2.3. Coexistence Scheme of Radar and Communication. In this section, we introduce the coexistence schemes of radar and communication, i.e., the ISFB and MUFB schemes, as shown in Figure 2.

The ISFB scheme is depicted in Figure 2(a). In this scheme, the total $K$ subcarriers are partitioned into two disjoint categories: the $K_{r}$ radar-only subcarriers and the $K_{c}$ communication-only subcarriers; then, we have

$$
B=K_{r} \Delta f+K_{c} \Delta f
$$

where $B$ denotes the bandwidth of the total available band. Clearly, the signals of radar and communication are transmitted on different subcarriers and can be processed independently.

The MUFB scheme is depicted in Figure 2(b). In this scheme, the occupied bandwidth of radar and communication bands satisfies $B \leq\left(K_{r}+K_{c}\right) \Delta f \leq 2 B$ in this scheme. The $K_{c}$ subcarriers are split into two categories: the $K_{1}$ communication-only subcarriers where only communication signal exists and $K_{2}$ mix-used subcarriers where both radar and communication signals exist $\left(K_{1}+K_{2}=K_{c}\right)$. Combing equation (13), the values of $K_{1}$ and $K_{2}$ can be, respectively, given by

$$
\begin{aligned}
& K_{1}=K-\frac{c}{2 \Delta R \Delta f}, \\
& K_{2}=\frac{c}{2 \Delta R \Delta f}+K_{c}-K .
\end{aligned}
$$




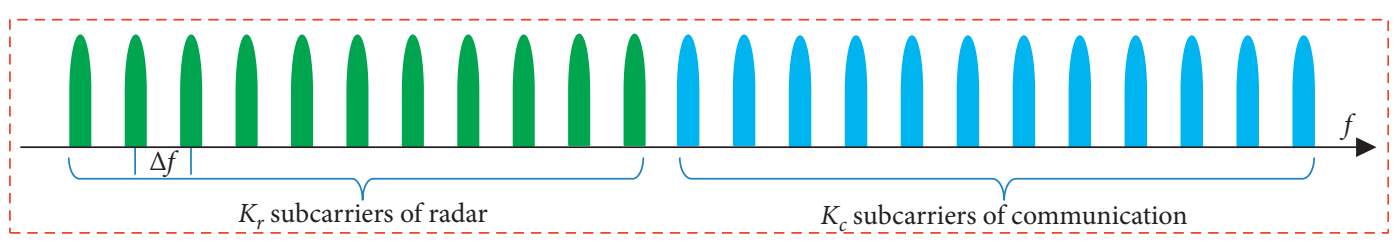

(a)

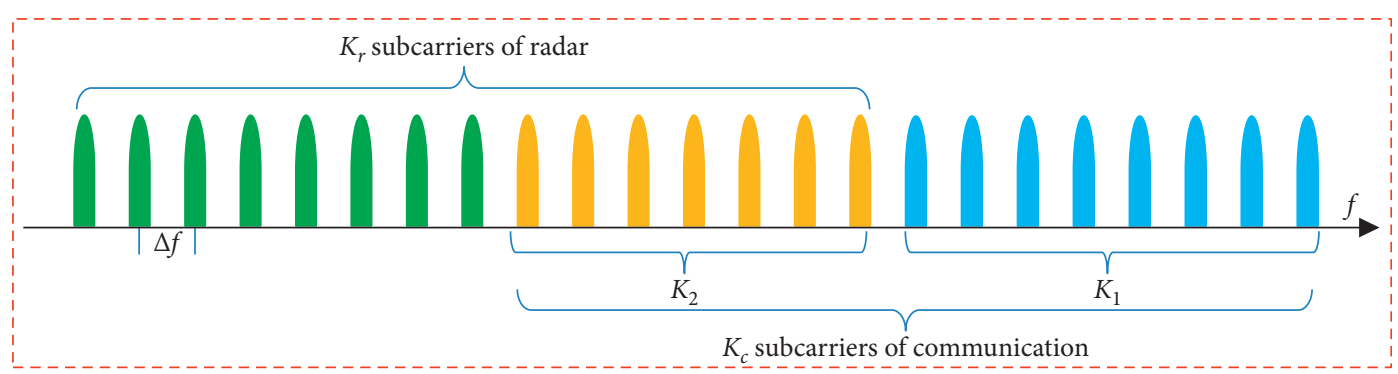

(b)

FIgURE 2: The coexistence schemes of radar and communication. (a) ISFB scheme; (b) MUFB scheme.

That is, radar and communication signals can be transmitted on the same subcarriers. The communication data rate $(\mathrm{CDR})$ is maximized with the interference from the radar, which is determined by the range resolution and scanning speed.

By comparing Figures 2(a) and 2(b), we can see that the ISFB scheme can be regarded as a special case of the MUFB scheme. However, we still study both schemes because, in the ISFB scheme, we can treat radar and communication systems independently from a signal processing point of view. This makes the ISFB is easy to handle and more appealing for practical implementation. For the MUFB scheme, the performance of data transmission can be enhanced at the price of higher complexity (due to the additional interference to be handled).

\section{Data Transmission of ISFB Scheme}

In this section, we analyze the performance of data transmission of the ISFB scheme. In this scheme, part of the subcarriers is assigned to the radar and the rest is used by the communication so that the received signals of radar and communication can be processed independently.

3.1. Radar Echo Data. In this section, we analyze the amount of RED under a certain range resolution $\Delta R$ and scanning speed $f_{\theta}$. Since the radar receiver is turned off during pulse transmission, the minimum detection range $R_{\min }$ can be given by [38]

$$
R_{\min }=\frac{c T_{p}}{2}
$$

where $c$ denotes the speed of light and $T_{p}$ is the pulse duration. Under a certain detection probability $p_{d}$, the maximum detection range $R_{\max }$ can be given by [38]

$$
R_{\max }\left(f_{\theta}\right)=\left(\frac{\operatorname{tr}\left(\widetilde{S} \widetilde{S}^{H}\right) \tau A_{e} \sigma}{4 \pi k_{0} T_{0} D_{0}(1) T_{p r i}} \frac{1}{f_{\theta}}\right)^{1 / 4}
$$

where $D_{0}(1)$ denotes the detection factor, $\sigma$ is target cross section, $T_{\text {pri }}$ denotes the pulse repetition interval (PRI), and $k_{0}, T_{0}$, and $A_{e}$ denote Boltzmann constant, absolute temperature, and antenna effective area, respectively. The received radar signals are sampled beginning at $t_{1}=2 R_{\min } / c$ and ending at $t_{2}=2 R_{\max }\left(f_{\theta}\right) / c+T_{p}$. According to the Nyquist sampling theorem, the sampling interval $T_{\text {sam }}$ should not be larger than $1 /\left(K_{r} \Delta f\right)$, and for convenience, we adopt $T_{\text {sam }}=1 /\left(K_{r} \Delta f\right)$ in this paper, which means the sampling number $N_{s}$ equals to the number of range cell, that is,

$$
N_{s}=\frac{R_{\max }\left(f_{\theta}\right)-R_{\min }}{\Delta R},
$$

and for a chirp signal, we have

$$
\Delta R=\frac{c}{2 K_{r} \Delta f}
$$

The number of pulse transmitted in 1 second is

$$
n_{p}=\frac{1}{T_{p r i}}
$$

The amount of radar echo data in 1 second can be expressed as

$$
\mathscr{D}\left(\Delta R, f_{\theta}\right)=N_{s} N_{\mathrm{bit}} n_{p}=\frac{\left(R_{\mathrm{max}}\left(f_{\theta}\right)-R_{\mathrm{min}}\right) N_{\mathrm{bit}}}{\Delta R T_{\mathrm{pri}}}\left(\frac{\mathrm{bit}}{\mathrm{s}}\right),
$$

where $N_{\text {bit }}$ denotes the quantify bits without apparent saturation.

From (15), we can find that, with fixed PRI $T_{\text {pri }}$, pulse duration $T_{p}$, and quantify bit $N_{\text {bit }}$, the amount of RED 
$\mathscr{D}\left(\Delta R, f_{\theta}\right)$ is determined by range resolution $\Delta R$ and maximum detection range $R_{\max }$. The smaller $\Delta R$ (or larger $\left.R_{\max }\right)$ is, the larger amount of $\mathscr{D}\left(\Delta R, f_{\theta}\right)$ is. Moreover, the smaller $f_{\theta}$ is, the larger $R_{\max }$ is. Therefore, there will be a larger $\mathscr{D}\left(\Delta R, f_{\theta}\right)$ with a smaller $f_{\theta}$.

3.2. Communication Data Rate for ISFB Scheme. Since radar and communication signals are operated on the isolated subcarriers in the ISFB scheme, the total communication power $P_{c}$ is allocated to the $K_{c}$ subcarriers according to the corresponding channel conditions without interference from the radar system. Let $\mathbf{S}_{c} \in C^{K_{c} \times M}$ be the transmitted communication waveform matrix for the ISFB scheme, i.e., $\mathbf{S}_{c}=\left[\mathbf{s}(1), \mathbf{s}(2), \ldots, \mathbf{s}\left(K_{c}\right)\right]^{T}$. Since there is no interference from radar system, the CDR for the ISFB scheme can be given by

$$
\mathscr{C}_{I}=K_{c} \Delta f\left[\log _{2} \operatorname{det}\left(\mathbf{S}_{c} \Sigma \mathbf{S}_{c}^{H}+\Sigma_{\mathbf{w}}\right)-\log _{2} \operatorname{det}\left(\Sigma_{\mathbf{w}}\right)\right]\left(\frac{\text { bit }}{\mathrm{s}}\right) .
$$

Then, with the constraint of total communication power $P_{c}$, the maximization problem of CDR can be formulated as

$$
\begin{array}{ll}
\max _{\mathbf{S}_{c}} & \log _{2} \operatorname{det}\left(\mathbf{S}_{c} \Sigma \mathbf{S}_{c}^{H}+\Sigma_{\mathbf{w}}\right)-\log _{2} \operatorname{det}\left(\Sigma_{\mathbf{w}}\right), \\
\text { s.t. } & \operatorname{tr}\left(\mathbf{S}_{c} \mathbf{S}_{c}^{H}\right) \leq P_{c},
\end{array}
$$

where the constant multiplier $K_{c} \Delta f$ is omitted. Since $\log _{2} \operatorname{det}\left(\Sigma_{\mathbf{w}}\right)$ does not depend on the transmitted waveform $\mathbf{S}_{c}$, problem (17) is equivalent to the following problem:

$$
\begin{array}{ll}
\max _{\mathbf{S}_{c}} & \log _{2} \operatorname{det}\left(\mathbf{S}_{c} \Sigma \mathbf{S}_{c}^{H}+\Sigma_{\mathbf{w}}\right), \\
\text { s.t. } & \operatorname{tr}\left(\mathbf{S}_{c} \mathbf{S}_{c}^{H}\right) \leq P_{c} .
\end{array}
$$

To obtain the optimal solution of (18), a lemma is firstly introduced.

Lemma 1 (see [39]). Define $\mathbf{A}$ and $\mathbf{B}$ as $N \times N$ positive semidefinite Hermitian matrices with eigendecomposition $\mathbf{A}=\mathbf{U}_{\mathbf{A}} \mathbf{R}_{\mathbf{A}} \mathbf{U}_{\mathbf{A}}^{H}$ and $\mathbf{B}=\mathbf{U}_{\mathbf{B}} \mathbf{R}_{\mathbf{B}} \mathbf{U}_{\mathbf{B}}^{H}$, respectively, the eigenvalues of $\mathbf{A}$ and $\mathbf{B}$ satisfy that $\lambda_{1} \geq \lambda_{2} \geq \cdots \geq \lambda_{N}$ and $\iota_{1} \geq \iota_{2} \geq \cdots \geq \iota_{N}$, and then we have

$$
\prod_{n=1}^{N}\left(\lambda_{n}+\iota_{n}\right) \leq \operatorname{det}(\mathbf{A}+\mathbf{B}) \leq \prod_{n=1}^{N}\left(\lambda_{n}+\iota_{N+1-n}\right),
$$

where the lower bound is derived if and only if $\mathbf{U}_{\mathbf{A}}=\mathbf{U}_{\mathbf{B}}$ while the upper bound is derived if and only if $\mathbf{U}_{\mathbf{A}}=\mathbf{U}_{\mathbf{B}} \mathbf{T}$ with $\mathbf{T}$ denoting the permutation matrix:

$$
\mathbf{T}=\left[\begin{array}{cccc}
0 & 0 & \cdots & 1 \\
0 & \cdots & 1 & 0 \\
\vdots & 1 & \vdots & \vdots \\
1 & 0 & \cdots & 0
\end{array}\right]
$$

Since $\mathbf{S}_{c} \Sigma \mathbf{S}_{c}^{H}$ and $\Sigma_{\mathbf{w}}$ are positive semidefinite Hermitian matrices, the corresponding eigendecomposition can be computed by

$$
\begin{aligned}
\mathbf{S}_{c} \Sigma \mathbf{S}_{c}^{H} & =\mathbf{U}_{0} \mathbf{R}_{0} \mathbf{U}_{0}^{H}, \\
\Sigma_{\mathbf{w}} & =\mathbf{V}_{\mathbf{w}} \mathbf{R}_{\mathbf{w}} \mathbf{V}_{\mathbf{w}}^{H},
\end{aligned}
$$

where $\mathbf{R}_{0}=\operatorname{diag}\left(\zeta_{0,1}, \zeta_{0,2}, \ldots, \zeta_{0, K}\right), \zeta_{0,1} \geq, \ldots, \geq \zeta_{0, K_{c}}$ and $\mathbf{R}_{\mathbf{w}}=\operatorname{diag}\left(\zeta_{\mathbf{w}, 1}, \zeta_{\mathbf{w}, 2}, \ldots, \zeta_{\mathbf{w}, K_{c}}\right), \zeta_{\mathbf{w}, 1} \geq, \ldots, \geq \zeta_{\mathbf{w}, K_{c}}$. According to Lemma 1 , we have

$$
\log _{2} \operatorname{det}\left(\mathbf{S}_{c} \Sigma \mathbf{S}_{c}^{H}+\Sigma_{\mathbf{w}}\right) \geq \log _{2} \prod_{k=1}^{K}\left(\zeta_{0, k}+\zeta_{\mathbf{w}, k}\right),
$$

with equality achieved at $\mathbf{U}_{0}=\mathbf{V}_{\mathbf{w}} \mathbf{T}$.

Let the eigendecomposition of $\Sigma$ be

$$
\Sigma=\mathbf{V}_{1} \mathbf{R}_{\Sigma} \mathbf{V}_{1} \text {, }
$$

where $\mathbf{R}_{\Sigma}=\operatorname{diag}\left(\zeta_{\Sigma, 1}, \ldots, \zeta_{\Sigma, K_{c}}\right)$. Therefore, the optimal solution of problem (18) can be obtained by solving

$$
\begin{aligned}
\max _{\zeta_{s, k}} & \sum_{k=1}^{K_{c}} \log _{2}\left(\zeta_{s, k} \zeta_{\Sigma, k}+\zeta_{\mathbf{w}, k}\right), \\
\text { s.t. } & \sum_{k=1}^{K_{c}} \zeta_{s, k} \leq P_{c},
\end{aligned}
$$

where $\zeta_{s, k}$ is the $k$-th diagonal element of $\mathbf{S}_{c} \mathbf{S}_{c}^{H}$.

Since optimization problem (24) is convex, the optimal solution of which can be characterized by using the Karush-Kuhn-Tucker (KKT) optimality conditions [40, 41]. The corresponding Lagrangian form of the above optimization problem is

$$
\begin{aligned}
\mathscr{L}\left(\zeta_{s, k}, \mu_{k}, \nu\right)= & -\sum_{k=1}^{K_{c}} \log _{2}\left(\zeta_{s, k} \zeta_{\Sigma, k}+\zeta_{\mathrm{w}, k}\right) \\
& -\mu_{k} \sum_{k=1}^{K_{c}} \zeta_{s, k}+\nu\left(\sum_{k=1}^{K_{c}} \zeta_{s, k}-P_{c}\right),
\end{aligned}
$$

where $\mu_{k}$ and $\nu$ are Lagrangian multipliers. Then, the optimal solution $\zeta_{s, k}^{*}$ can be obtained, i.e.,

$$
\zeta_{s, k}=\left(\frac{1}{v}-\frac{\zeta_{\mathrm{w}, k}}{\zeta_{\Sigma, k}}\right)^{+}
$$

where $v$ can be obtained by solving

$$
\sum_{k=1}^{K_{c}}\left(\frac{1}{v}-\frac{\zeta_{\mathrm{w}, k}}{\zeta_{\Sigma, k}}\right)^{+}=P_{c}
$$

3.3. Data Throughput for ISFB Scheme. Data throughput for the integrated system is a parameter characterizing the maximum data rate, which is determined by the smaller of CDR and RED. Therefore, define $\mathscr{F}_{I}$ as the data throughput for the ISFB scheme, and we have 


$$
\mathscr{J}_{I}\left(\Delta R, f_{\theta}\right)=\min \left\{\mathscr{C}_{I}, \mathscr{D}\left(\Delta R, f_{\theta}\right)\right\},\left(\frac{\text { bit }}{s}\right),
$$

where $\mathscr{D}\left(\Delta R, f_{\theta}\right)$ is given in equation (15). On one hand, $\mathscr{F}_{I}=D\left(\Delta R, f_{\theta}\right)$ means that the CDR $\mathscr{C}_{I}$ can meet the requirement of data transmission and the RED can be transmitted timely and there is no transmission delay. On the other hand, $\mathscr{J}_{I}=\mathscr{C}_{I}$ means the amount of radar echo data $\mathscr{D}\left(\Delta f, f_{\theta}\right)$ is larger than the $\mathrm{CDR} \mathscr{C}_{I}$, the integrated system needs several PRIs to transmit all the received radar echo data, and the corresponding transmission delay $\mathscr{T}_{I}$ is defined as

$$
\mathscr{T}_{I}=\left(\frac{\mathscr{D}\left(\Delta R, f_{\theta}\right)-\mathscr{J}_{I}\left(\Delta R, f_{\theta}\right)}{\mathscr{J}_{I}\left(\Delta R, f_{\theta}\right)}, 0\right)^{+} .
$$

\section{Data Transmission of MUFB Scheme}

In this section, we analyze the performance of data transmission for the MUFB scheme. Since the amount of RED related to the range resolution $\Delta R$ and scanning speed $f_{\theta}$ is the same for the ISFB and MUFB schemes, the description is not given again. In this scheme, radar and communication signals can be used on the same subcarriers. The CDR is maximized along with the interference from the radar, which is determined by the range resolution and scanning speed.

4.1. Communication Data Rate for MUFB Scheme. Let $\widetilde{\mathbf{S}}_{r} \in C^{K_{2} \times M}$ be the transmitted radar waveform matrix on the $K_{2}$ mix-used subcarriers, i.e., $\widetilde{\mathbf{S}}_{r}=\left[\mathbf{s}(1), \mathbf{s}(2), \ldots, \mathbf{s}\left(K_{2}\right)\right]^{T}$. Then, from the communication system's point of view, the interference matrix $\Gamma \in C^{K_{c} \times K_{c}}$ caused by radar signal is

$$
\Gamma=\left[0_{K_{1} \times K_{1}}, \widetilde{\mathbf{S}}_{r} \Sigma_{r} \widetilde{\mathbf{S}}_{r}^{H}\right]^{T} .
$$

Therefore, the CDR of the MUFB scheme can be given by

$$
\begin{aligned}
\mathscr{C}_{M}= & K_{c} \Delta f\left(\log _{2} \operatorname{det}\left(\mathbf{S}_{c} \Sigma \mathbf{S}_{c}^{H}+\Gamma+\Sigma_{\mathbf{w}}\right)\right. \\
& \left.-\log _{2} \operatorname{det}\left(\Gamma+\Sigma_{\mathbf{w}}\right)\right)\left(\frac{\text { bit }}{s}\right) .
\end{aligned}
$$

Then, with the constraint of total transmitted communication power $P_{c}$, the maximization problem of CDR can be formulated as

$$
\begin{aligned}
& \max _{\mathbf{S}_{c}} \log _{2} \operatorname{det}\left(\mathbf{S}_{c} \Sigma \mathbf{S}_{c}^{H}+\Gamma+\Sigma_{\mathbf{w}}\right)-\log _{2} \operatorname{det}\left(\Gamma+\Sigma_{\mathbf{w}}\right), \\
& \text { s.t. } \operatorname{tr}\left(\mathbf{S}_{c} \mathbf{S}_{c}^{H}\right) \leq P_{c},
\end{aligned}
$$

where we have omitted the constant multiplier $K_{c} \Delta f$. Since $\left.\log _{2} \operatorname{det}\left(\Gamma+\Sigma_{\mathbf{w}}\right)\right)$ does not depend on the transmitted waveform $\mathbf{S}_{c}$, the optimal solution of problem (32) can be obtained by solving

$$
\begin{aligned}
& \max _{\mathbf{S}_{c}} \log _{2} \operatorname{det}\left(\mathbf{S}_{c} \Sigma \mathbf{S}_{c}^{H}+\Gamma+\Sigma_{\mathbf{w}}\right), \\
& \text { s.t. } \operatorname{tr}\left(\mathbf{S}_{c} \mathbf{S}_{c}^{H}\right) \leq P_{c} .
\end{aligned}
$$

Define $\eta=\operatorname{diag}\left\{\eta_{1}, \ldots, \eta_{K_{c}}\right\}$ as the diagonal elements of interference matrix $\Gamma$, and the interference from the radar system to the communication system can be given in the following proposition.

proposition 1. Let the eigendecompositions of $\widetilde{\Sigma}_{H}$ and $\Sigma_{r}$ be

$$
\widetilde{\Sigma}_{H}=\widetilde{\mathbf{V}}_{1} \mathbf{R}_{H} \widetilde{\mathbf{V}}_{1},
$$

and

$$
\Sigma_{r}=\mathbf{V}_{r} \mathbf{R}_{r} \mathbf{V}_{r}
$$

where $\mathbf{R}_{H}=\operatorname{diag}\left(\widetilde{\xi}_{H, 1}, \ldots, \widetilde{\xi}_{H, K_{r}}\right)$ and $\mathbf{R}_{r}=\operatorname{diag}\left(\zeta_{r, 1}, \ldots\right.$, $\left.\zeta_{r, K_{c}}\right)$. Then, the interference from the radar system on the $k$-th subcarrier can be given by

$$
\eta_{k}= \begin{cases}0, & 1 \leq k \leq K_{1}, \\ \widetilde{\xi}_{s, \tilde{K}-k}^{*} \zeta_{r, k}, & K_{1}+1 \leq k \leq K_{c},\end{cases}
$$

where $\widetilde{K}=K_{r}+K_{1}+1$ and $\widetilde{\xi}_{\tilde{S}-k}$ denotes the optimal allocated radar power on the $k$-th subcarrier given by

$$
\widetilde{\xi}_{s, \tilde{K}-k}^{*}=\left(\frac{1}{\widetilde{v}}-\frac{\widetilde{\xi}_{\mathbf{w}, \widetilde{K}-k}}{\widetilde{\xi}_{H, \widetilde{K}-k}}\right)^{+} .
$$

Proof. See Appendix.

As a result, let $\varsigma_{s, k}$ be the $k$-th diagonal element of $\mathbf{S}_{c} \mathbf{S}_{c}^{H}$ for the MUFB scheme, the optimal power allocation solution $\varsigma_{s, k}^{*}$ can be obtained by solving the following problem:

$$
\begin{aligned}
& \max _{\varsigma_{s, k}} \sum_{k=1}^{K_{c}} \log _{2}\left(\varsigma_{s, k} \zeta_{\Sigma, k}+\eta_{k}+\zeta_{\mathbf{w}, k}\right), \\
& \text { s.t. } \quad \sum_{k=1}^{K_{c}} \varsigma_{s, k} \leq P_{c} .
\end{aligned}
$$

Similar to the ISFB scheme, we solve the above problem by Lagrange multipliers and the corresponding Lagrange form is needed, namely,

$$
\begin{aligned}
\mathscr{L}\left(\varsigma_{s, k}, \mu_{k}, \lambda\right)= & -\sum_{k=1}^{K_{c}} \log _{2}\left(\varsigma_{s, k} \zeta_{\Sigma, k}+\eta_{k}+\zeta_{\mathrm{w}, k}\right) \\
& -\mu_{k} \sum_{k=1}^{K_{c}} \varsigma_{s, k}+\lambda\left(\sum_{k=1}^{K_{c}} \varsigma_{s, k}-P_{c}\right),
\end{aligned}
$$

where $\mu_{k}$ and $\lambda$ are Lagrangian multipliers. Then, the optimal solution $\varsigma_{s, k}^{*}$ can be obtained, i.e.,

$$
\zeta_{s, k}=\left(\frac{1}{\lambda}-\frac{\eta_{k}+\zeta_{\mathbf{w}, k}}{\zeta_{\Sigma, k}}\right)^{+}
$$

where $v$ can be obtained by solving 


$$
\sum_{k=1}^{K_{c}}\left(\frac{1}{v}-\frac{\eta_{k}+\zeta_{\mathrm{w}, k}}{\zeta_{\Sigma, k}}\right)^{+}=P_{c}
$$

4.2. Data Throughput for MUFB Scheme. Similar to the ISFB scheme, the data throughput for the MUFB scheme is determined by the smaller of $\operatorname{CDR} \mathscr{C}_{M}$ and $\operatorname{RED} \mathscr{D}\left(\Delta R, f_{\theta}\right)$, that is,

$$
\mathscr{J}_{M}\left(\Delta R, f_{\theta}\right)=\min \left\{\mathscr{C}_{M}, \mathscr{D}_{J}\left(\Delta R, f_{\theta}\right)\right\},\left(\frac{\text { bit }}{s}\right),
$$

where $\mathscr{D}\left(\Delta R, f_{\theta}\right)$ is given in equation (15). On one hand, when $\mathscr{J}_{M}=\mathscr{D}\left(\Delta R, f_{\theta}\right)$, the RED $\mathscr{D}$ can be transmitted timely without transmission delay. On the other hand, when $\mathscr{J}_{M}=\mathscr{C}_{M}$, there will be transmission delay, which can be defined as

$$
\mathscr{T}_{M}=\left(\frac{\mathscr{D}\left(\Delta R, f_{\theta}\right)-\mathscr{J}_{M}\left(\Delta R, f_{\theta}\right)}{\mathscr{J}_{M}\left(\Delta R, f_{\theta}\right)}, 0\right)^{+} .
$$

\section{Numerical Examples}

In this section, we provide numerical examples to demonstrate the performance of data transmission for the JRCS under different values of range resolution $\Delta R$ and scanning speed $f_{\theta}$. Also, for comparison, the simulated results of average power allocation (APA) are presented (APA implies that the total power is allocated across all the available subcarriers). The performance of the proposed adaptive optimization algorithm and that of the APA $\underset{\widetilde{\xi}}{\text { are labeled as }}$ "AOA" and "APA," respectively. We assume $\widetilde{\xi}_{H, k}, \zeta_{r, k}$, and $\zeta_{\Sigma, k}$ obey

$$
\begin{gathered}
\widetilde{\xi}_{H, k}=\beta_{H, k} \frac{G_{r}^{2} \lambda_{r, k}^{2}}{(4 \pi)^{3} R_{r t}^{4}}, \quad 1 \leq k \leq K_{r}, \\
\zeta_{r, k}=\beta_{r, k} \frac{G_{r} G_{c} \lambda_{r, k}^{2}}{(4 \pi)^{3} R_{r t}^{2} R_{t c}^{2}}, \quad 1 \leq k \leq K_{c}, \\
\zeta_{\Sigma, k}=\beta_{\Sigma, k} \frac{G_{c}^{2} \lambda_{c, k}^{2}}{(4 \pi)^{3} R_{r c}^{2}}, \quad 1 \leq k \leq K_{c},
\end{gathered}
$$

where $G_{r}$ and $G_{c}$ denote antenna gains of radar and communication, respectively; $\lambda_{r, k}$ and $\lambda_{c, k}$ denote wavelengths of radar and communication on the $k$-th subcarrier, respectively; $R_{r t}$ ranges between the integrated transmitter and interest of target; $R_{t c}$ ranges between interest of target and communication receiver (without loss of generality, we assume $\left.R_{r t}=R_{t c}=R_{\max }\right) ; R_{r c}$ ranges between the integrated transmitter and communication receiver; $\beta_{H, k}, \beta_{r, k}$, and $\beta_{\Sigma, k}$ are generated from Gaussian distributions $\mathscr{C} \mathscr{N} \sim\left(0, \sigma_{H, k}^{2}\right)$, $\mathscr{C} \mathcal{N} \sim\left(0, \sigma_{r, k}^{2}\right)$, and $\mathscr{C} \mathcal{N} \sim\left(0, \sigma_{\Sigma, k}^{2}\right)$, respectively, where $\sigma_{H, k}^{2}, \sigma_{r, k}^{2}$, and $\sigma_{\Sigma, k}^{2}$ are set to be 1 in this simulation. Without no explicit specification, the setting of other system parameters is given in Table 1 .
TABLE 1: Simulation parameters.

\begin{tabular}{lc}
\hline Parameter & Value \\
\hline False alarm probability $\left(p_{f}\right)$ & $1 \times 10^{-5}$ \\
Bandwidth $(B)$ & $100 \mathrm{MHz}$ \\
System noise temperature $\left(T_{\text {sys }}\right)$ & $1000 \mathrm{~K}$ \\
Detection transmit power $\left(P_{r}\right)$ & $100 \mathrm{~kW}$ \\
Communication transmit power $\left(P_{c}\right)$ & $10 \mathrm{~W}$ \\
Communication gain $\left(G_{c}\right)$ & 100 \\
Communication range $\left(R_{r c}\right)$ & $10 \mathrm{~km}$ \\
Antenna effective area $\left(A_{e}\right)$ & $1 \mathrm{~m}^{2}$ \\
Pulse repetition interval $\left(T_{\text {pri }}\right)$ & $2 \mathrm{~ms}$ \\
Pulse width $\left(T_{p}\right)$ & $20 \mu \mathrm{s}$ \\
Boltzmann constant $\left(k_{0}\right)$ & $1.38 \times 10^{-23}$ \\
Subcarrier spacing & $0.5 \mathrm{MHz}$ \\
\hline
\end{tabular}

Figure 3 shows the maximum detection range $R_{\max }$ versus $f_{\theta}$ under $p_{d}=0.85,0.9$, and 0.95. As expected, a monotonic decreasing behavior of the maximum detection range $R_{\max }$ can be observed when the scanning speed $f_{\theta}$ is larger. Also, we can see that the $R_{\max }$ gets smaller as the $p_{d}$ gets larger. This is because a larger $p_{d}$ needs a larger detection factor, leading to a smaller $R_{\max }$.

Figure 4 depicts the amount of RED $\mathscr{D}$ for different values of $\Delta R$ and $f_{\theta} . \mathscr{D}$ is determined by sampling interval $T_{\text {sam }}$ and the number of sampling $N_{s}$, as discussed in Section 3.1. We can see that, on one hand, $\mathscr{D}$ is getting larger with the decrease of $\Delta R$. A smaller $\Delta R$ needs a larger size of the bandwidth of radar band leads to a smaller sampling interval $T_{\text {sam }}$ according to the Nyquist sampling theorem. Therefore, the smaller $\Delta R$ is, the larger $\mathscr{D}$ is. On the other hand, a smaller $f_{\theta}$ leads to a larger detection area, as shown in Figure 3, which will increase the number of sample $N_{s}$. Thus, there will be a larger $\mathscr{D}$ along with a smaller $f_{\theta}$.

Under $f_{\theta}=300 \% \mathrm{~s}, 200 \% \mathrm{~s}, 100 \% \mathrm{~s}$, and $50 \% \mathrm{~s}$ for both AOA and APA, the CDR for the ISFB and MUFB schemes versus range resolution $\Delta R$ is depicted in Figure 5:

(i) In Figure 5(a), a monotonic increasing behavior of the CDR for both AOA and APA can be observed as the range resolution $\Delta R$ gets larger. This is because the larger $\Delta R$ results in the smaller number of radar subcarrier $K_{r}$. Then, more subcarriers are used by the communication system, leading to a larger CDR $\mathscr{C}_{I}$. Also, the CDR in this scheme has no relationship with the scanning speed $f_{\theta}$, since radar and communication signals are operated on isolated subcarriers.

(ii) In Figure 5(b), a monotonic increasing behavior of the CDR for the MUFB scheme can be observed as the range resolution $\Delta R$ gets larger. The larger $\Delta R$ is, the smaller number of mix-used subcarrier $K_{2}$ is. Then, the smaller interference from the radar system to the communication system leads to a larger CDR $\mathscr{C}_{M}$. Also, we can notice that a smaller $f_{\theta}$ leads to a larger CDR $\mathscr{C}_{M}$ by reducing the received radar power. As a consequence, the smaller interference to communication leads to a higher $\operatorname{CDR} \mathscr{C}_{M}$.

(iii) By comparing Figures 5(a) and 5(b), the achieved CDR of the MUFB scheme is larger than the ISFB 


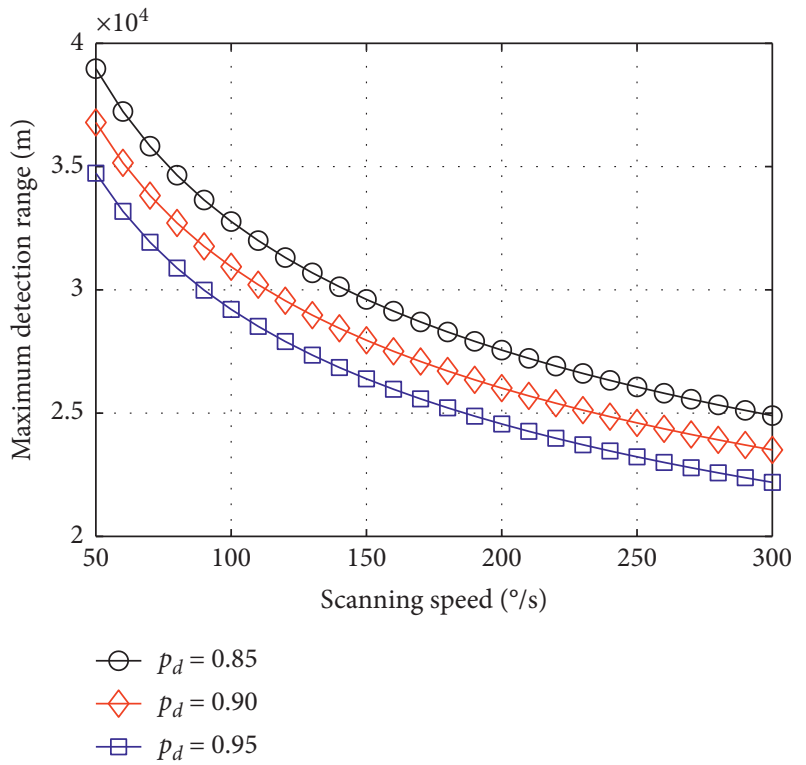

Figure 3: Maximum detection range $R_{\max }$ versus $f_{\theta}$ under $p_{d}=$ $0.85,0.9$, and 0.95 .

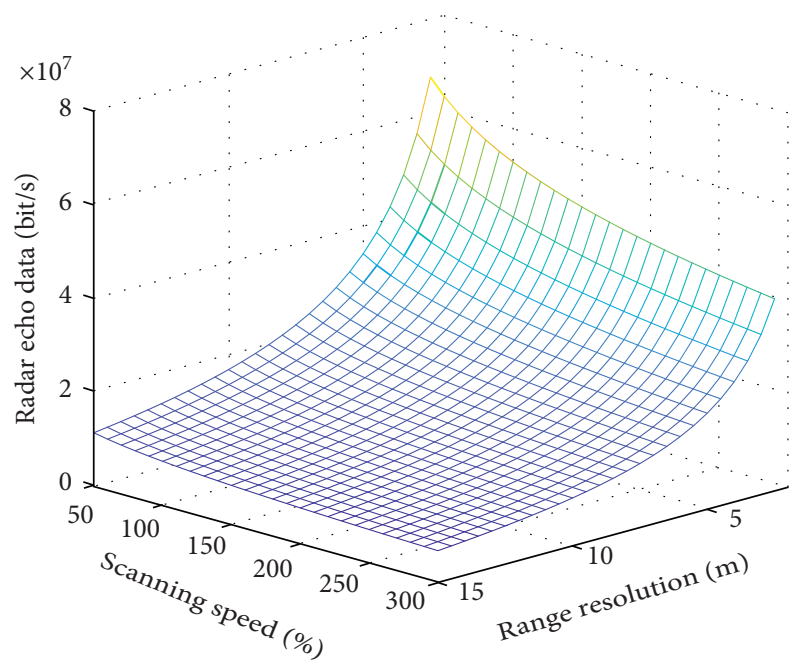

FIgURE 4: The amount of RED $\mathscr{D}$ versus range resolution $\Delta R$ and scanning speed $f_{\theta}$.

scheme. This is because all available subcarriers can be used by both systems in the MUFB scheme, which leads to an increase of CDR even there being interference from cofrequency radar signal. Also, by comparing the AOA and APA, a significant gain in the CDR can be achieved by employing the AOA. This is because, when the AOA is employed, the transmit power is allocated based on the conditions of subcarriers, which enable significantly maximizing the CDR performance; however, the transmit power is averagely distributed among the available subcarriers when the AOA is employed.

For both AOA and APA, Figure 6 depicts the data throughput for different values of $\Delta R$ under $f_{\theta}=300^{\circ} / \mathrm{s}$ : (i) For the MUFB scheme, the achieved maximum data throughput is $2.36 \times 10^{7} \mathrm{bit} / \mathrm{s}$ corresponding to $\Delta R=4 \mathrm{~m}$. In the case of $\Delta R \leq 4 \mathrm{~m}$, the amount of RED $\mathscr{D}$ is larger than the CDR $C_{M}$; then, the data throughput $\mathscr{T}_{M}$ is determined by $\mathrm{CDR} \mathscr{C}_{M}$, which cannot meet the requirement of data transmission and leads to data retention. However, in the case of $\Delta R \geq 4 \mathrm{~m}$, the CDR $\mathscr{C}_{M}$ is larger than the amount of RED $\mathscr{D}$ and the data throughput $\mathscr{F}_{M}$ is determined by $\mathscr{D}$, which means the RED can be transmitted timely.

(ii) For the ISFB scheme, the maximum data throughput is $1.59 \times 10^{7} \mathrm{bit} / \mathrm{s}$ corresponding to $\Delta R=6 \mathrm{~m}$. The data through $\mathscr{J}_{I}$ is determined by the amount of RED $\mathscr{D}$ when $\Delta \leq 6 \mathrm{~m}$ and the CDR $\mathscr{C}_{I}$ when $\Delta \geq 6 \mathrm{~m}$.

(iii) We can see that the maximum data throughput of the MUFB scheme is larger than that of the ISFB scheme, i.e., the performance of the MUFB scheme is better. By comparing Figures 6(a) and 6(b), a significant gain in the data throughput can be achieved by employing the AOA. This is because, when the $\mathrm{AOA}$ is employed, the values of $\mathrm{CDR}$ are much larger, as shown in Figure 5. To give further insight, the data throughput with different values of scanning speed $f_{\theta}$ is then discussed.

Figure 7 depicts the data throughput versus range resolution $\Delta R$ under $f_{\theta}=300(\%), 200(\% / \mathrm{s}), 100(\% / \mathrm{s})$, and $50(\%)$ :

(i) The simulated results of the ISFB scheme employing AOA are depicted in Figure 7(a), and points $A_{1}, B_{1}$, $C_{1}$, and $D_{1}$ correspond to the maximum data throughput $\mathscr{F}_{I}$ of the ISFB scheme for $f_{\theta}=300(\mathrm{o} / \mathrm{s})$, $200(\% / \mathrm{s}), 100(\% / \mathrm{s})$, and $50(\%)$, respectively. We can see that the maximum data throughput is getting smaller with the increase of $f_{\theta}$. This is because the larger $f_{\theta}$ is, the smaller amount of RED is, leading a smaller maximum data throughput.

(ii) The simulated results of the MUFB scheme employing AOA are depicted in Figure 7(b), and points $A_{2}, B_{2}, C_{2}$, and $D_{2}$ correspond to the maximum data throughput $\mathscr{F}_{M}$ of the MUFB scheme for $f_{\theta}=300(\% / s), 200(\% / s), 100(\% / s)$, and $50(\%)$, respectively. By comparing Figures $7(\mathrm{a})$ and 7 (b), in the case of large $\Delta R$ (such as $\Delta R \geq 10 \mathrm{~m}$ ), the data throughput of both schemes are the same, which is because the data throughput is determined by the amount of RED, as discussed in Figure 6 . Meanwhile, in the case of small $\Delta R$ (such as $\Delta R \leq 6 \mathrm{~m}$ ), the data throughput of the MUFB scheme is larger since a larger CDR of the MUFB scheme; that is, the performance of the MUFB scheme is much better than that of the ISFB scheme.

(iii) The simulated results of the ISFB and MUFB schemes employing APA are depicted in Figures $7(\mathrm{c})$ and $7(\mathrm{~d})$, respectively. By comparing the simulated results depicted in Figures 7(a) and 


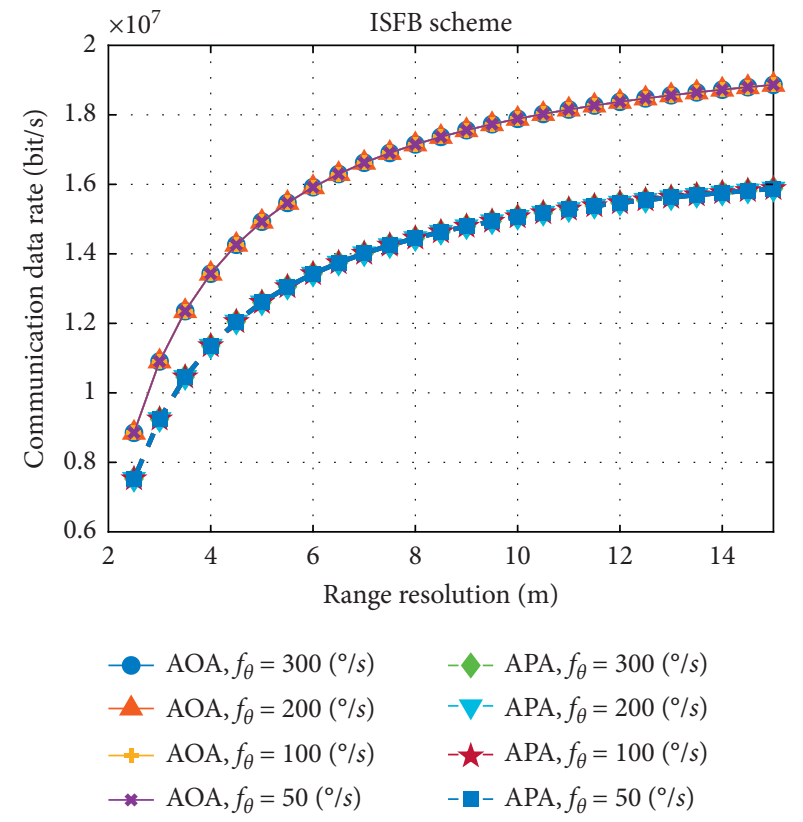

(a)

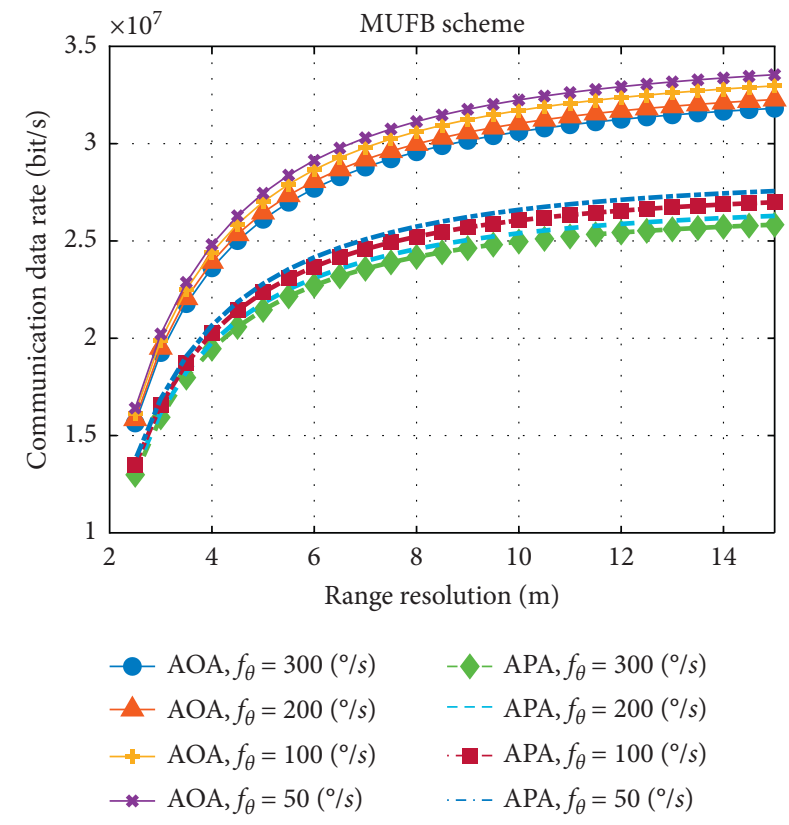

(b)

Figure 5: CDR versus range resolution $\Delta R$ under $f_{\theta}=300 \%$ s, $200 \%$ s, $100 \%$, and $50 \%$ s. (a) ISFB scheme; (b) MUFB scheme.

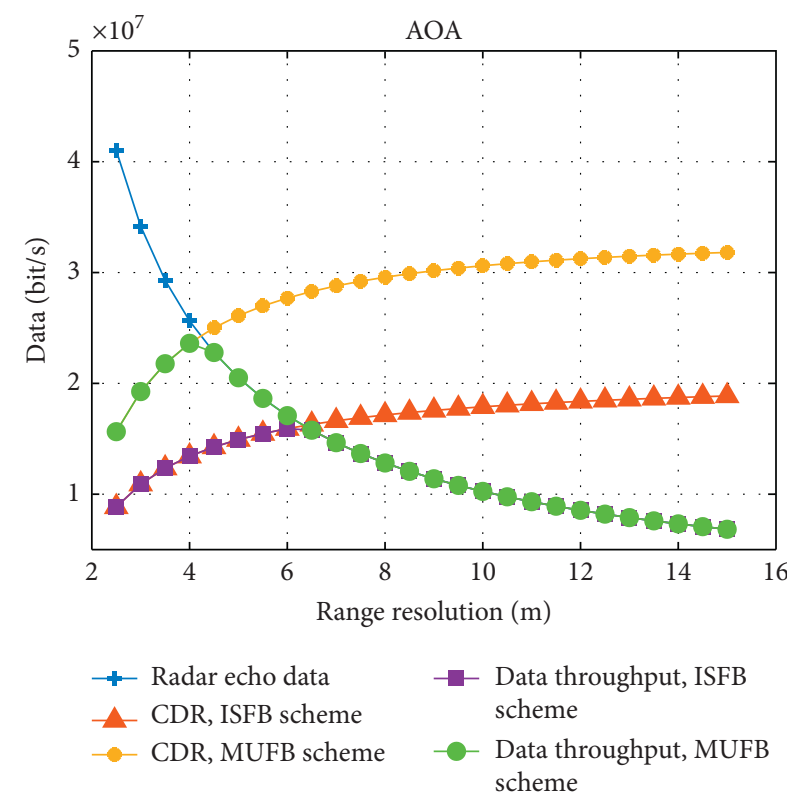

(a)

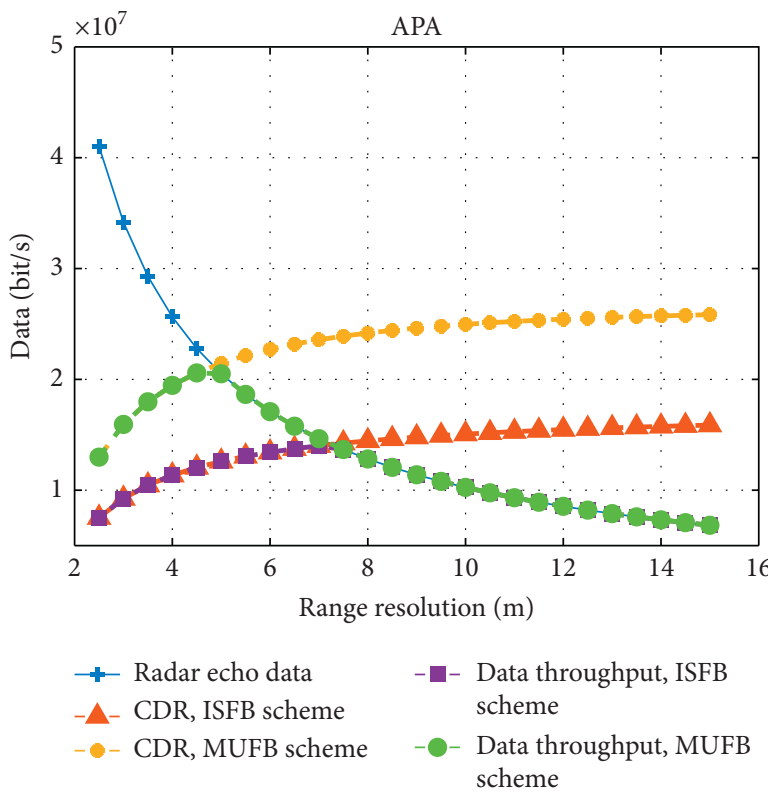

(b)

FIGURE 6: RED, CDR, and the corresponding data throughput for different values of range resolution $\Delta R$ with $f_{\theta}=300^{\circ} / \mathrm{s}$. (a) AOA; (b) APA.

7(b), we can see that a significant gain in the data throughput can be achieved by employing the AOA This is because, when the AOA is employed, the values of $\mathrm{CDR}$ are much larger, as shown in Figure 5.
Figure 8 depicts the range resolution $\Delta R$ corresponding to the maximum data throughput for different values of $f_{\theta}$ for both schemes. A nonincreasing behavior can be observed when the scanning speed $f_{\theta}$ is larger. This is because the data throughput is determined by $f_{\theta}$ and $\Delta R$, the larger $f_{\theta}$ (or 


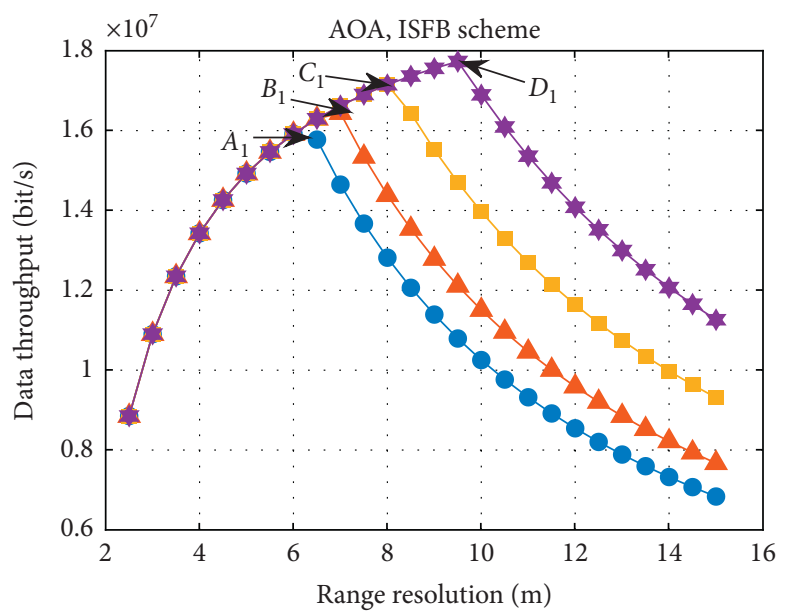

$$
\begin{array}{ll}
\longrightarrow f_{\theta}=300(\%) & -f_{\theta}=100(\% / s) \\
-f_{\theta}=200(\% / s) & -f_{\theta}=50(\% / s)
\end{array}
$$

(a)

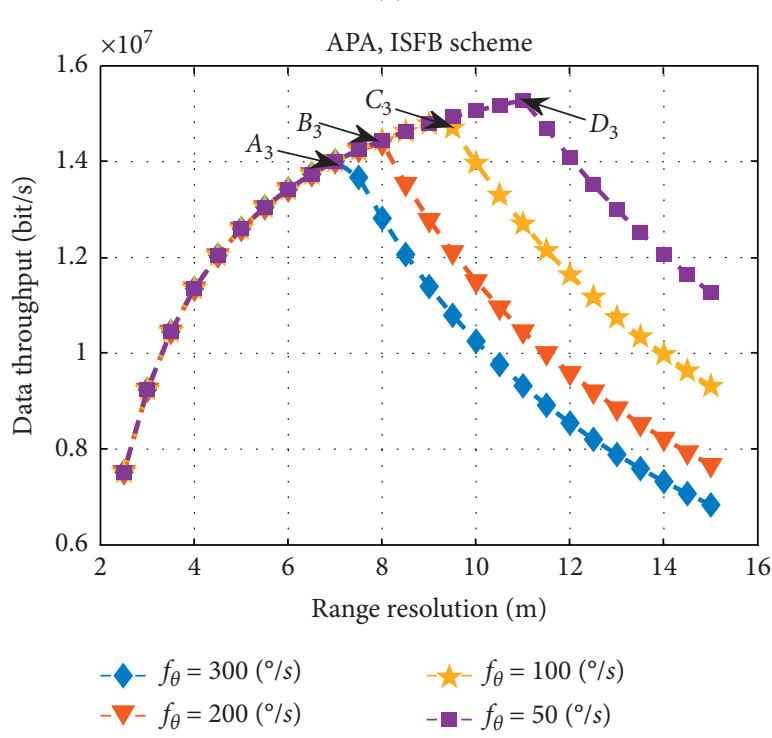

(c)

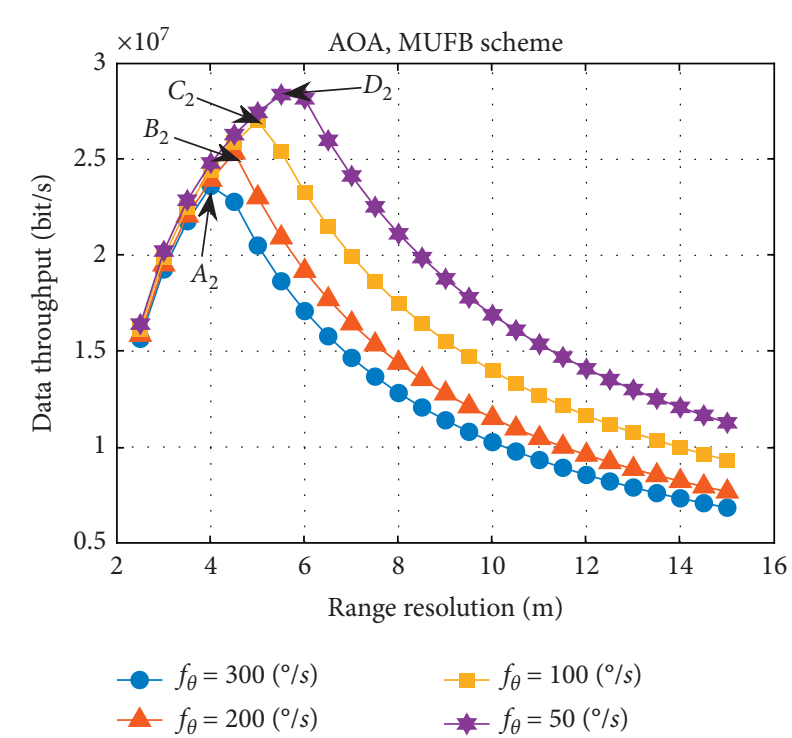

(b)

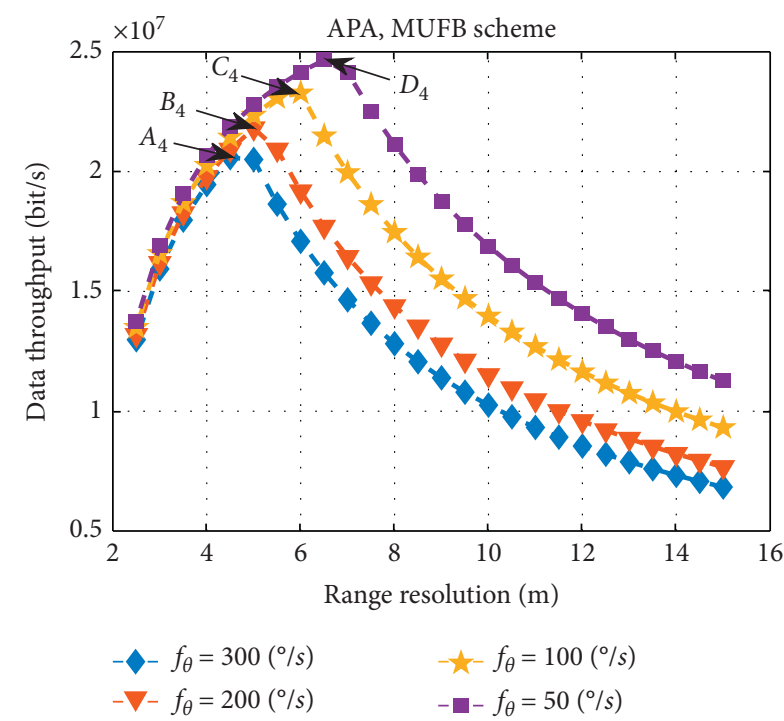

(d)

FIGURE 7: Data throughput versus range resolution $\Delta R$ under $f_{\theta}=300(\%), 200(\%), 100(\%)$, and $50(\%)$ ). (a) The ISFB scheme employing AOA; (b) the MUFB scheme employing AOA; (c) the ISFB scheme employing APA; (d) the MUFB scheme employing APA.

$\Delta R)$ the smaller the data throughput. Thus, with a certain requirement of maximum data throughput, a smaller $\Delta R$ can be obtained with the increase of $f_{\theta}$. We can see that the $\Delta R$ of the MUFB scheme is smaller than that of the ISFB scheme, which means that the performance of the MUFB scheme is better. This is because, as mentioned earlier, the CDR of the MUFB scheme is much larger than that of the ISFB scheme, which can meet a higher requirement of data transmission (corresponds to smaller $\Delta R$ ). Also, take the MUFB scheme as an example, the range resolution employing $\mathrm{AOA}$ is much smaller than that employing APA, which implies the AOA case can achieve better performance.
Figure 9 shows the delay time for different values of $\Delta R$ under $f_{\theta}=300 \% \mathrm{~s}, 200 \% \mathrm{~s}, 100 \%$, and $50 \%$ s. A monotonic decreasing behavior of delay time can be observed for all schemes. It is because that the amount of RED is getting smaller and CDR is getting larger with the increase of $\Delta R$. For $f_{\theta}=300 \%, 200 \%, 100 \%$, and $50 \%$, the delay time of the MUFB scheme is smaller than the ISFB scheme so that the performance of the MUFB scheme is better. For all cases, under a certain $\Delta R$, the delay time is the largest when $f_{\theta}=50 \%$ s. It is because a smaller $f_{\theta}$ causes a smaller amount of data throughput corresponding to a larger delay time. Also, for all cases, the delay time employing APA is 


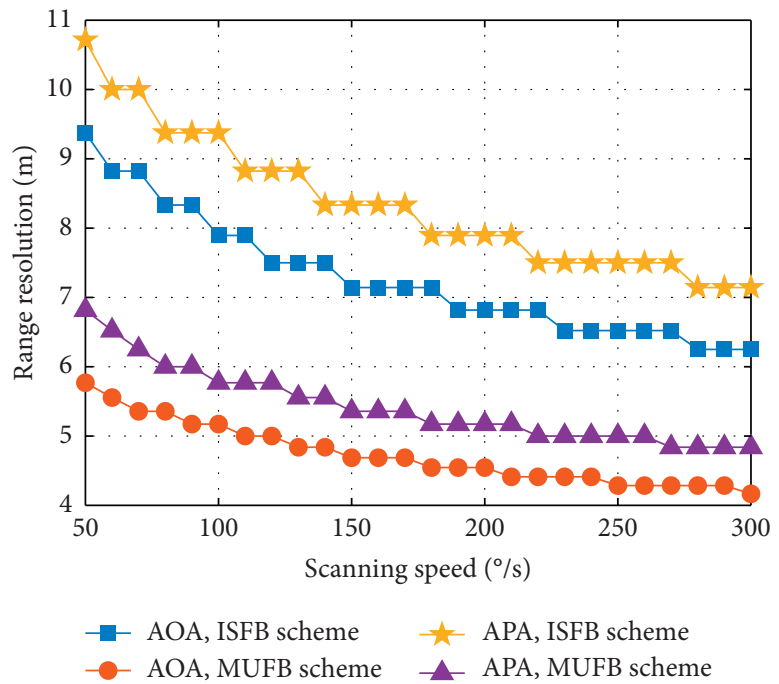

FIGURE 8: Range resolution $\Delta R$ corresponding to the maximum data throughput versus scanning speed $f_{\theta}$.
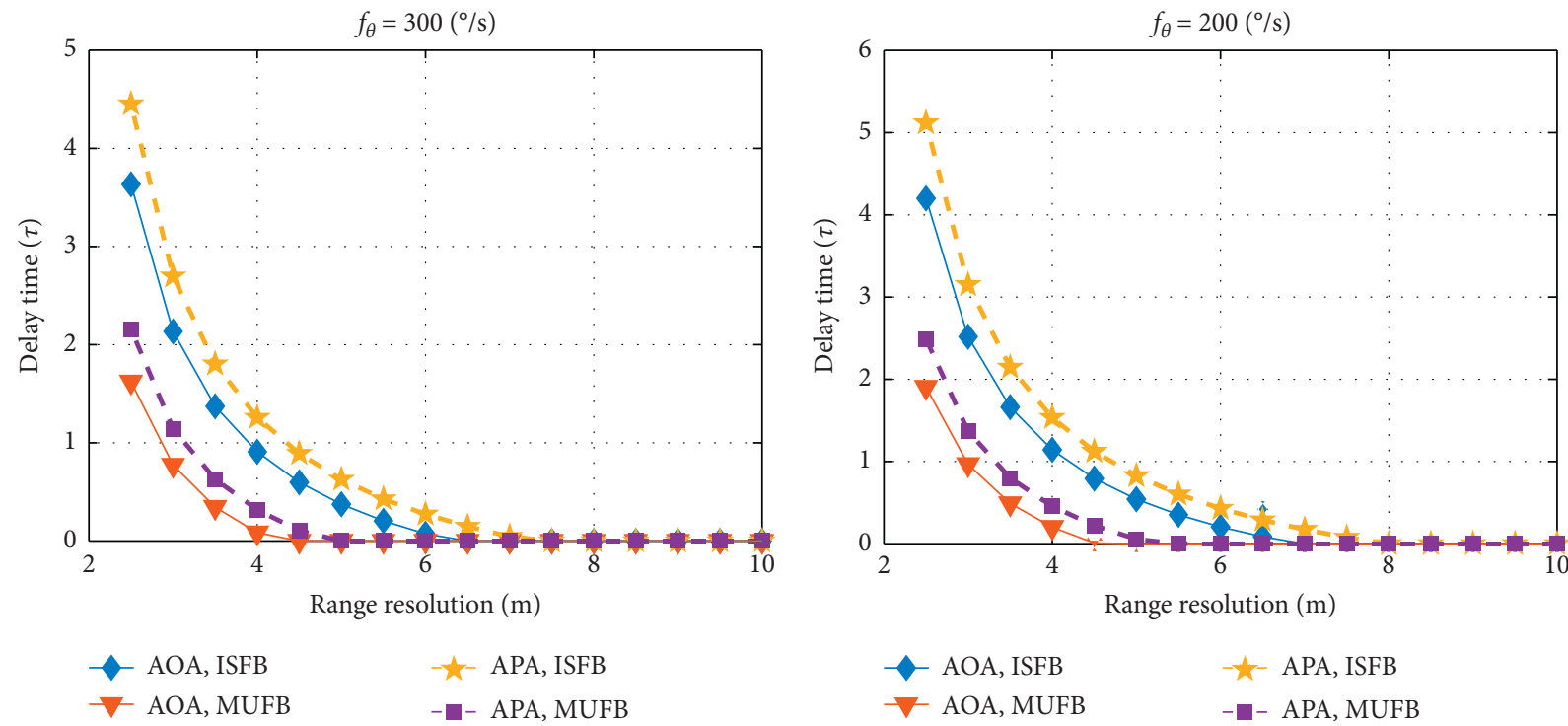

(a)

(b)
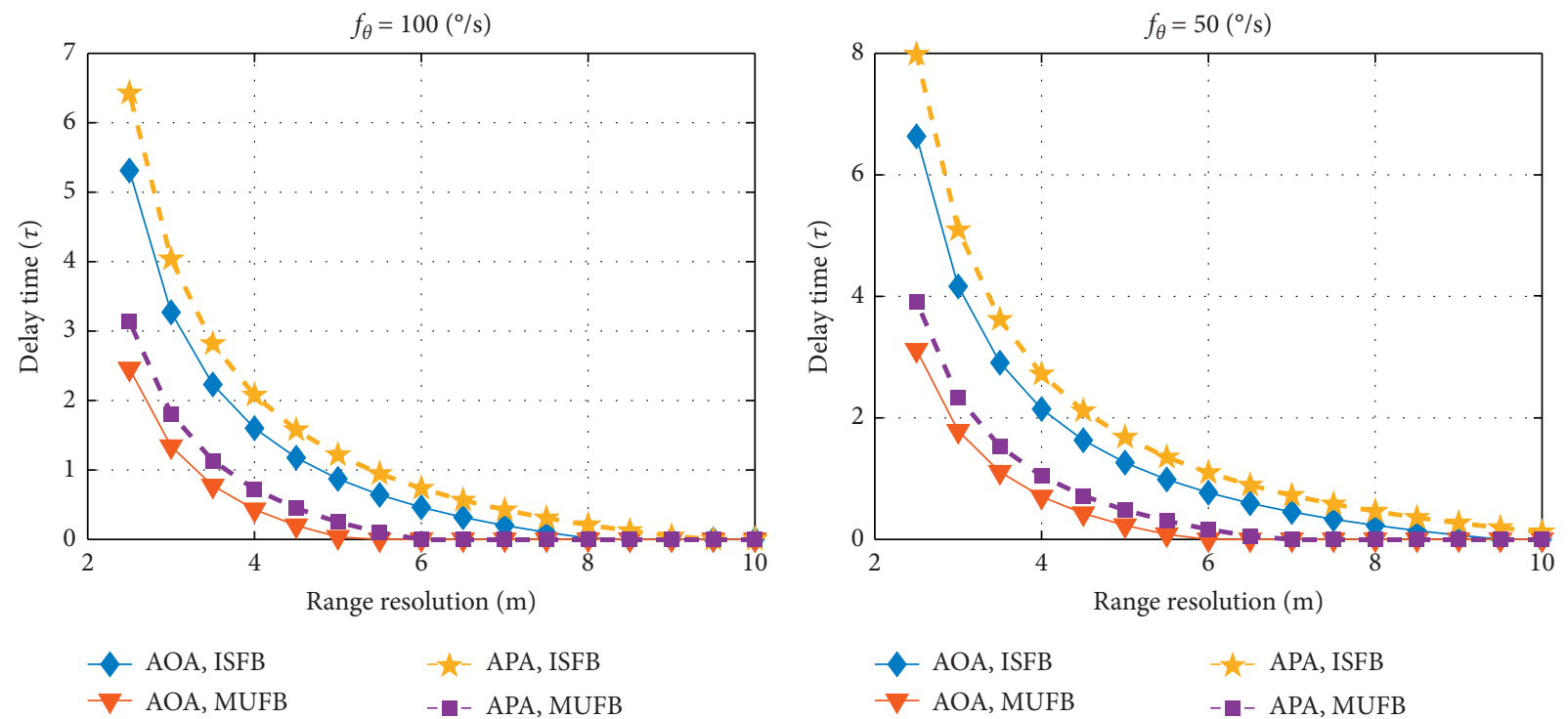

(c)

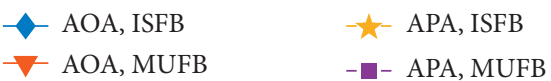

(d)

Figure 9: Delay time versus range resolution $\Delta R$. (a) $f_{\theta}=300 \%$; (b) $f_{\theta}=200 \%$; (c) $f_{\theta}=100 \%$; (d) $f_{\theta}=50 \%$ s. 
much smaller than that employing AOA, which implies the former has a better performance.

\section{Conclusion}

In this context, the performance of data transmission for the JRCS has been evaluated. Two spectral coexistence schemes are, respectively, proposed. The first one named the ISFB scheme processes the radar and communication signals independently. The other one, named the MUFB scheme, allows the radar and communication signals to operate on the same subcarriers. Comparing the two schemes, we can find the ISFB scheme brings the advantage of easy execution and interference avoidance while the MUFB scheme significantly improves the performance of data transmission over the ISFB scheme by maximizing the CDR with the presence of radar interference and meanwhile obtaining the optimal power allocation solution of radar power via maximizing radar mutual information. Simulation results have shown the performance of the data transmission for the JRCS, and a significant performance gain in the data transmission can be achieved, compared to the APA case.

\section{Appendix}

Proof. with the constraint of total radar power $P_{r}$, the optimal allocation solutions of radar, $\widetilde{\xi}_{s, k}^{*}$, can be obtained by solving the following problem:

$$
\begin{aligned}
& \max _{\mathbf{S}_{c}} \log _{2} \operatorname{det}\left(\widetilde{\mathbf{S}} \widetilde{\boldsymbol{\Sigma}}_{H} \widetilde{\mathbf{S}}^{H}+\widetilde{\Sigma}_{\mathbf{w}}\right)-\log _{2} \operatorname{det}\left(\widetilde{\Sigma}_{\mathbf{w}}\right), \\
& \text { s.t. } \operatorname{tr}\left(\widetilde{\mathbf{S}} \widetilde{\mathbf{S}}^{H}\right) \leq P_{r} .
\end{aligned}
$$

Since $\log _{2} \operatorname{det}\left(\widetilde{\Sigma}_{\mathbf{w}}\right)$ does not depend on the transmitted waveform $\widetilde{\mathbf{S}}$, the optimal solutions of problem (A.1) can be obtained by solving

$$
\begin{aligned}
& \max _{\mathbf{S}_{c}} \log _{2} \operatorname{det}\left(\widetilde{\mathbf{S}} \widetilde{\boldsymbol{\Sigma}}_{H} \widetilde{\mathbf{S}}^{H}+\widetilde{\Sigma}_{\mathbf{w}}\right) \\
& \text { s.t. } \operatorname{tr}\left(\widetilde{\mathbf{S}} \widetilde{\mathbf{S}}^{H}\right) \leq P_{r} .
\end{aligned}
$$

Since $\widetilde{\mathbf{S}} \widetilde{\Sigma}_{H} \widetilde{\mathbf{S}}^{H}$ and $\widetilde{\Sigma}_{\mathbf{w}}$ are positive semidefinite Hermitian matrices, the corresponding eigendecomposition can be given by

$$
\begin{aligned}
\widetilde{\mathbf{S}} \widetilde{\Sigma}_{H} \widetilde{\mathbf{S}}^{H} & =\widetilde{\mathbf{U}}_{0} \widetilde{\mathbf{R}}_{0} \widetilde{\mathbf{U}}_{0}^{H}, \\
\widetilde{\Sigma}_{\mathbf{w}} & =\widetilde{\mathbf{V}}_{\mathbf{w}} \widetilde{\mathbf{R}}_{\mathbf{w}} \widetilde{\mathbf{V}}_{\mathbf{w}}^{H},
\end{aligned}
$$

respectively, where $\widetilde{\mathbf{R}}_{0}=\operatorname{diag}\left(\widetilde{\xi}_{Q 1}, \widetilde{\xi}_{0,2}, \ldots, \widetilde{\xi}_{0, K_{r}}\right), \widetilde{\xi}_{0,1} \geq, \ldots$, $\geq \underline{\xi}_{0, K_{r}}$ and $\widetilde{\mathbf{R}}_{\mathbf{w}}=\operatorname{diag}\left(\widetilde{\xi}_{\mathbf{w}, 1}, \widetilde{\xi}_{\mathbf{w}, 2}, \ldots, \widetilde{\xi}_{\mathbf{w}, K_{r}}\right), \widetilde{\xi}_{\mathbf{w}, 1} \geq, \ldots$, $\geq \widetilde{\xi}_{\mathbf{w}, K_{r}}$. Therefore, according to Lemma 1, problem (A.2) is equivalent to the following optimization problem:

$$
\begin{aligned}
& \max _{\widetilde{\xi}_{s, k}} \sum_{k=1}^{K_{r}} \log _{2}\left(\widetilde{\xi}_{s, k} \widetilde{\xi}_{H, k}+\widetilde{\xi}_{\mathrm{w}, k}\right) \\
& \text { s.t. } \quad \sum_{k=1}^{K_{r}} \widetilde{\xi}_{s, k} \leq P_{r},
\end{aligned}
$$

where $\widetilde{\xi}_{s, k}$ is the $k$-th diagonal element of $\widetilde{\mathbf{S}} \widetilde{\mathbf{S}}^{H}$. The optimal solutions of the above convex problem can be characterized by the KKT conditions, and the corresponding Lagrangian form is needed, namely,

$$
\begin{aligned}
\mathscr{L}\left(\xi_{s, k}, \tilde{\mu}\right)= & -\sum_{k=1}^{K_{r}} \log _{2}\left(\widetilde{\xi}_{s, k} \widetilde{\zeta}_{H, k}+\widetilde{\zeta}_{\mathrm{w}, k}\right) \\
& -\widetilde{\mu}_{k} \sum_{k=1}^{K_{r}} \widetilde{\xi}_{s, k}+\widetilde{v}\left(\sum_{k=1}^{K_{r}} \widetilde{\xi}_{s, k}-P_{r}\right),
\end{aligned}
$$

where $\tilde{\mu}_{k}$ and $\widetilde{\nu}$ are Lagrangian multipliers. Then, the KKT conditions are given as follows:

$$
\begin{aligned}
-\frac{\widetilde{\xi}_{H, k}}{\widetilde{\xi}_{s, k} \widetilde{\xi}_{H, k}+\widetilde{\xi}_{w, k}}-\widetilde{\mu}_{k}+\widetilde{v} & =0, \\
\tilde{\mu}_{k} \widetilde{\xi}_{s, k} & =0, \\
\widetilde{\xi}_{s, k} \geq 0, & \\
\sum_{k=1}^{K_{r}} \widetilde{\xi}_{s, k}-P_{r} & =0,
\end{aligned}
$$

$$
\tilde{\mu}_{k} \geq 0 .
$$

Eliminating $\widetilde{\mu}_{k}$, we have

$$
\begin{aligned}
v-\frac{\widetilde{\xi}_{H, k}}{\widetilde{\xi}_{s, k} \widetilde{\xi}_{H, k}+\widetilde{\xi}_{\mathbf{w}, k}} \geq 0, & \\
\mu_{k}\left(\widetilde{v}-\frac{\widetilde{\xi}_{H, k}}{\widetilde{\xi}_{s, k} \widetilde{\xi}_{H, k}+\widetilde{\xi}_{w, k}}\right) & =0, \\
\widetilde{\xi}_{s, k} & \geq 0, \\
\sum_{k=1}^{K_{r}} \widetilde{\xi}_{s, k}-P_{c} & =0 .
\end{aligned}
$$

Then, the optimal solution $\widetilde{\xi}_{s, k}^{*}$ can be given by

$$
\widetilde{\xi}_{s, k}^{*}=\left(\frac{1}{\bar{v}}-\frac{\widetilde{\xi}_{\mathrm{w}, k}}{\widetilde{\xi}_{H, k}}\right)^{+},
$$

where $\widetilde{v}$ can be obtained by solving

$$
\sum_{k=1}^{K_{r}}\left(\frac{1}{\bar{v}}-\frac{\widetilde{\xi}_{\mathbf{w}, k}}{\widetilde{\xi}_{H, k}}\right)^{+}=P_{r} .
$$


For the $K_{1}$ communication-only subcarriers, there is no radar signal, $\eta_{k}=0$, with $k=1, \ldots, K_{1}$; meanwhile, for the $K_{2}$ mix-used subcarriers, we have

$$
\eta_{k}=\widetilde{\xi}_{s, K_{r}+K_{1}-k+1}^{*} \widetilde{\xi}_{H, K_{r}+K_{1}-k+1},
$$

for $k=K_{1}+1, \ldots, K_{c}$, where $\widetilde{\xi}_{s, K_{r}+K_{1}-k+1}^{*}$ is given in equation (A.9). Till now, the result of equation (36) can be obtained.

\section{Data Availability}

The data used to support the findings of this study are available from the corresponding author upon request.

\section{Conflicts of Interest}

The authors declare that they have no conflicts of interest.

\section{References}

[1] T. Zhang, J. Liang, Y. Yang, G. Cui, L. Kong, and X. Yang, "Antenna deployment method for multistatic radar under the situation of multiple regions for interference," Signal Processing, vol. 143, pp. 292-297, 2018.

[2] X. Li, Y. Han, Z. Wang et al., "Fast antenna deployment method for multistatic radar with multiple dynamic surveillance regions," Signal Processing, vol. 170, pp. 107-419, 2020.

[3] T. Tian, X. Du, and G. Li, "Cramer-rao bounds of localization estimation for integrated radar and communication system," IEEE Access, vol. 8, pp. 105852-105863, 2020.

[4] J. Zheng, J. Li, H. Shi, Q. Liu, and X. Yang, "Joint subcarrier, code, and power allocation for parallel multi-radio access in heterogeneous wireless networks," Science China Information Sciences, vol. 57, no. 8, pp. 1-5, 2014.

[5] C. Zhang, J. Ge, J. Li, and Y. Hu, "Robust power allocation algorithm for analog network coding with imperfect CSI," Science China Information Sciences, vol. 57, no. 4, pp. 1-10, 2014.

[6] T. Tian, T. Zhang, and L. Kong, "Timeliness constrained task scheduling for multifunction radar network," IEEE Sensors Journal, vol. 19, pp. 525-534, 2018.

[7] L. Zheng, M. Lops, Y. C. Eldar, and X. Wang, "Radar and communication coexistence: an overview: a review of recent methods," IEEE Signal Processing Magazine, vol. 36, no. 5, pp. 85-99, 2019.

[8] J. Qian, M. Lops, L. Le Zheng, X. Wang, and Z. He, "Joint system design for coexistence of MIMO radar and MIMO communication," IEEE Transactions on Signal Processing, vol. 66, no. 13, pp. 3504-3519, 2018.

[9] H. Griffiths, L. Cohen, S. Watts et al., "Radar spectrum engineering and management: technical and regulatory issues," Proceedings of the IEEE, vol. 103, pp. 85-102, 2014.

[10] C. Baylis, M. Fellows, L. Cohen, and R. J. Marks II, "Solving the spectrum crisis: intelligent, reconfigurable microwave transmitter amplifiers for cognitive radar," IEEE Microwave Magazine, vol. 15, no. 5, pp. 94-107, 2014.

[11] A. Hassanien, M. G. Amin, E. Aboutanios, and B. Himed, "Dual-Function Radar Communication Systems: a solution to the spectrum congestion problem," IEEE Signal Processing Magazine, vol. 36, no. 5, pp. 115-126, 2019.
[12] A. R. Chiriyath, S. Ragi, H. D. Mittelmann, and D. W. Bliss, "Radar waveform optimization for joint radar communications performance," Electronics, vol. 8, no. 12, p. 1498, 2019.

[13] B. J. Donnet and I. D. Longstaff, "Combining MIMO radar with OFDM communications," in Proceedings of the $3 \mathrm{rd}$ European Radar Conference, pp. 37-40, Manchester UK, 2006.

[14] C. Sturm, T. Zwick, and W. Wiesbeck, "An OFDM system concept for joint radar and communications operations," in Proceedings of IEEE 69th Vehicular Technology Conference, pp. 1-5, Barcelona, Spain, 2009.

[15] C. Sturm and W. Wiesbeck, "Waveform design and signal processing aspects for fusion of wireless communications and radar sensing," Proceedings of the IEEE, vol. 99, no. 7, pp. 1236-1259, 2011.

[16] J. Euziere, R. Guinvarc'h, M. Lesturgie et al., "Dual function radar communication time-modulated array," in Proceedings of International Radar Conference, pp. 1-4, Washington, DC, 2014.

[17] A. Hassanien, M. G. Amin, Y. D. Zhang, and F. Ahmad, "Signaling strategies for dual-function radar communications: an overview," IEEE Aerospace and Electronic Systems Magazine, vol. 31, no. 10, pp. 36-45, 2016.

[18] A. Hassanien, M. G. Amin, Y. D. Zhang, and F. Ahmad, "Phasemodulation based dual-function radar-communications," IET Radar, Sonar \& Navigation, vol. 10, no. 8, pp. 1411-1421, 2016.

[19] A. Hassanien, M. G. Amin, Y. D. Zhang et al., "Dual-function radar-communications: information embedding using sidelobe control and waveform diversity," IEEE Transactions on Signal Processing, vol. 64, pp. 2168-2181, 2015.

[20] T. Tian, G. Li, and T. Zhou, "Power distribution for an OFDM-based dual-function Radar-Communication sensor," IEEE Sensors Letters, vol. 4, no. 11, pp. 1-4, 2020.

[21] M. Labib, V. Marojevic, A. F. Martone et al., "Coexistence between communications and radar systems: a survey," URSI Radio Science Bulletin, vol. 2017, pp. 74-82, 2017.

[22] A. Lackpour, M. Luddy, and J. Winters, "Overview of interference mitigation techniques between WiMAX networks and ground based radar," in Proceedings of 20th Annual Wireless and Optical Communications Conference (WOCC), pp. 1-5, Newark, NJ, USA, 2011.

[23] H. Deng and B. Himed, "Interference mitigation processing for spectrum-sharing between radar and wireless communications systems," IEEE Transactions on Aerospace and Electronic Systems, vol. 49, no. 3, pp. 1911-1919, 2013.

[24] A. R. Chiriyath, B. Paul, G. M. Jacyna et al., "Inner bounds on performance of radar and communications co-existence," IEEE Transactions on Signal Processing, vol. 64, pp. 464-474, 2015.

[25] B. Paul, A. R. Chiriyath, and D. W. Bliss, "Joint communications and radar performance bounds under continuous waveform optimization: the waveform awakens," in Proceedings of IEEE Radar Conference (RadarConf), pp. 1-6, Atlanda, GA, USA, 2016.

[26] A. Khawar, A. Abdelhadi, and C. Clancy, "Target detection performance of spectrum sharing MIMO radars," IEEE Sensors Journal, vol. 15, no. 9, pp. 4928-4940, 2015.

[27] P. Kumari, J. Choi, N. Gonzðllez-Prelcic et al., "IEEE 802.11 ad-based radar: an approach to joint vehicular communication-radar system," IEEE Transactions on Vehicular Technology, vol. 67, pp. 3012-3027, 2017.

[28] Y. L. Sit, B. Nuss, and T. Zwick, "On mutual interference cancellation in a MIMO OFDM multiuser radar-communication network," IEEE Transactions on Vehicular Technology, vol. 67, pp. 3339-3348, 2017. 
[29] B. Li, A. P. Petropulu, and W. Trappe, "Optimum co-design for spectrum sharing between matrix completion based MIMO radars and a MIMO communication system," IEEE Transactions on Signal Processing, vol. 64, no. 17, pp. 4562-4575, 2016.

[30] A. R. Al-Salehi, I. M. Qureshi, A. N. Malik, Z. Khan, and W. Khan, "Throughput enhancement for dual-function radarembedded communications using two generalized sidelobe cancellers," IEEE Access, vol. 7, pp. 91390-91398, 2019.

[31] C. Shi, F. Wang, M. Sellathurai et al., "Power minimization-based robust OFDM radar waveform design for radar and communication systems in coexistence," IEEE Transactions on Signal Processing, vol. 66, pp. 1316-1330, 2017.

[32] T. Tian, T. Zhang, G. Li, and T. Zhou, "Mutual information-based power allocation and co-design for multicarrier Radar and communication systems in coexistence," IEEE Access, vol. 7, pp. 159300-159312, 2019.

[33] F. Wang, H. Li, and M. A. Govoni, "Power allocation and co-design of multicarrier communication and radar systems for spectral coexistence," IEEE Transactions on Signal Processing, vol. 67, no. 14, pp. 3818-3831, 2019.

[34] Y. Liu, G. Liao, J. Xu, Z. Yang, and Y. Zhang, "Adaptive OFDM integrated radar and communications waveform design based on information theory," IEEE Communications Letters, vol. 21, no. 10, pp. 2174-2177, 2017.

[35] Z. Zhu, S. Kay, and R. S. Raghavan, "Information-theoretic optimal radar waveform design," IEEE Signal Processing Letters, vol. 24, no. 3, pp. 274-278, 2017.

[36] B. Tang, J. Tang, and Y. Peng, "MIMO radar waveform design in colored noise based on information theory," IEEE Transactions on Signal Processing, vol. 58, pp. 4684-4697, 2010.

[37] B. Tang and J. Li, "Spectrally constrained MIMO radar waveform design based on mutual information," IEEE Transactions on Signal Processing, vol. 67, pp. 821-834, 2018.

[38] M. I. Skolnik, Radar Handbook, McGraw-Hill, New York, NY, USA, 1990.

[39] M. Fiedler, "Bounds for the determinant of the sum of hermitian matrices," Proceedings of the American Mathematical Society, vol. 30, no. 1, p. 27, 1971.

[40] S. Boyd and L. Vandenberghe, Convex Optimization, Cambridge University Press, Cambridge, UK, 2004.

[41] D. Xu and Q. Li, "Effective capacity region and power allocation for two-way spectrum sharing cognitive radio networks," Science China Information Sciences, vol. 58, no. 6, pp. 1-10, 2015.

[42] T. M. Cover and J. A. Thomas, Elements of Information Theory, Wiley, Hoboken, NJ, USA, 2012. 